\title{
Evolução dos casos de HIV/AIDS em pessoas com 50 anos ou mais atendidos em um hospital universitário do nordeste brasileiro
}

\author{
Evolution of HIV/AIIDS cases in people aged 50 years and over attended at a university hospital in
}

the Northeast of Brazil

La evolución de los casos de VIH/SIDA en las personas de 50 años o mayores tratados en un hospital universitario en el Noreste de Brasil

Recebido: 12/03/2021 | Revisado: 19/03/2021 | Aceito: 22/03/2021 | Publicado: 31/03/2021

Linda Djeyme Santos
ORCID: https://orcid.org/0000-0001-7253-5424
Universidade Federal de Alagoas, Brasil
E-mail: linda-djeyme@hotmail.com
Amuzza Aylla Pereira dos Santos
ORCID: https://orcid.org/0000-0001-6299-7190
Universidade Federal de Alagoas, Brasil
E-mail: amuzza.santos@gmail.com
Isabel Comassetto
ORCID: https://orcid.org/0000-0002-2389-9384
Universidade Federal de Alagoas, Brasil
E-mail: isabelcomassetto@gmail.com
Julio Cesar Silva Oliveira
ORCID: https://orcid.org/0000-0003-2267-9010
Universidade Federal de Alagoas, Brasil
E-mail: enf.juliocsoliveira@gmail.com
Wanderlei Barbosa dos Santos
ORCID: https://orcid.org/0000-0001-9813-8857
Universidade Federal de Alagoas, Brasil
E-mail: wanderlei.santos@esenfar.ufal.br
José Augustinho Mendes Santos
ORCID: https://orcid.org/0000-0002-1570-4102
Universidade Federal de Alagoas, Brasil
E-mail: augustinhomendes1@gmail.com

\begin{abstract}
Resumo
O presente estudo tem como objetivo analisar a evolução dos indivíduos acima de 50 anos ou mais com diagnóstico de infeção pelo Vírus da Imunodeficiência Humana/Síndrome de Imunodeficiência Adquirida (HIV/AIDS) atendidos em um Hospital universitário do Nordeste do Brasil, sendo um estudo epidemiológico descritivo, documental, com abordagem quantitativa, de uma série histórica no período de 2006 a 2016. Os dados foram coletados através da base de dados do núcleo de epidemiologia do hospital. O estudo demonstrou um aumento progressivo para evolução dos indivíduos com 50 anos de idade ou mais que apresentam o diagnóstico de HIV/AIDS, sendo os mais acometidos são os homens na faixa etária entre 50 a 59 anos, heterossexuais. Conclui-se que assistir aos indivíduos que estão em processo de envelhecimento, que se apresentam sexualmente ativos e cercados por preconceitos, exige a atitude de profissionais cada vez mais preparados, abertos a novos paradigmas e com conhecimento das particularidades apresentadas por eles.
\end{abstract}

Palavras-chave: HIV; Síndrome de Imunodeficiência Adquirida; Idoso.

\begin{abstract}
This study aims to analyze the evolution of individuals over 50 years of age or older diagnosed with infection by the Human Immunodeficiency Virus / Acquired Immunodeficiency Syndrome (HIV / AIDS) at a University Hospital in the Northeast of Brazil, this is a descriptive, documental epidemiological study with a quantitative approach, from a historical series from 2006 to 2016. The data were collected through the hospital epidemiology core database. The study showed a progressive increase in the evolution of individuals with 50 years of age or older who are diagnosed with HIV/AIDS, the most affected being men in the age group between 50 and 59 years, heterosexual. It is concluded that to assist individuals who are in the process of aging, who are sexually active and surrounded by prejudices, requires the attitude of professionals who are increasingly prepared, open to new paradigms and with knowledge of the particularities presented by them.
\end{abstract}


Keywords: HIV; Acquired Immunodeficiency Syndrome; Aged.

\section{Resumen}

El presente estudio tiene como objetivo analizar la evolución de los individuos mayores de 50 años de edad o mayores con un diagnóstico de Virus de Inmunodeficiencia Humana / Síndrome de Inmunodeficiencia Adquirida (VIH / SIDA) tratados en un Hospital Universitario del Noreste de Brasil, siendo un estudio epidemiológico descriptivo, documental, con un enfoque cuantitativo, a partir de una serie histórica para el período 2006 a 2016. Los datos fueron recolectados a través de la base de datos central epidemiología del hospital. El estudio demostró un aumento progresivo en la evolución de los individuos de 50 años o mau que tienen un diagnóstico de VIH / SIDA, siendo los más afectados los hombres en el grupo de edad entre 50 a 59 años, heterosexuales. Se concluye que ver a la gente que están envejeciendo, que tienen vida sexual activa y rodeado de prejuicios, requiere la actitud de los profesionales cada vez más cualificados, abiertos a nuevos paradigmas y el conocimiento de las peculiaridades presentadas por ellos.

Palabras clave: VIH; Síndrome de Inmunodeficiencia Adquirida; Anciano.

\section{Introdução}

O envelhecimento da população brasileira torna-se cada vez mais evidente e segundo a Projeção da População divulgada pelo Instituto Brasileiro de Geografia e Estatística (IBGE) no ano de 2018 o número de idosos brasileiros poderá ultrapassar os 30 milhões (IBGE, 2019). No que se refere à saúde, sabe-se que os indivíduos com idade mais elevada apresentam progressão para a imunossupressão de forma mais acelerada, observando-se, ainda, maior aparecimento de doenças oportunistas, acrescido de maior probabilidade do advento de outras comorbidades, o que contribui para o agravamento das condições de saúde (Lima \& Souza, 2018; Pourcher, Goumelen, Bureau \& Bouee, 2020).

Paralelamente, a epidemia de HIV/Aids vem sofrendo diversas modificações em seu perfil ao longo do tempo, dentre as quais os fenômenos de feminilização, heterossexualização, adolescência, pauperização e envelhecimento. Estas características referenciam que não existem mais indivíduos particularmente vulneráveis ao vírus HIV, já que todas as fases do ciclo de vida estão expostas à contaminação, evidenciando uma epidemia de múltiplas dimensões que, ao longo do tempo, tem apresentado consideráveis transformações em sua evolução e distribuição (Suto et al., 2020).

Desta forma, junta-se a problemática do processo de envelhecimento da população brasileira, repleta de agravos sociais, econômicos e sanitários, à emergência das infecções ou doenças sexualmente transmissíveis, incluindo a AIDS, refletindo assim em um aumento do número de casos de HIV na população idosa (Andrade, Ayres, Alencar, Duarte, \& Parada, 2017).

O progresso de envelhecimento das pessoas que vivem com HIV/AIDS no Brasil e no mundo deu destaque a diversas questões relacionadas à saúde do idoso, dentre elas: a abordagem da sexualidade na fase do pré-envelhecimento, o uso de drogas e os direitos humanos, o acesso aos serviços e insumos de prevenção, entre tantas outras que, muitas vezes, não são abordadas (Silva et al., 2018).

De acordo com um estudo realizado pela Organização Mundial de Saúde (OMS) (2009), evidenciou-se a impossibilidade de se avaliar a realidade dos casos de HIV/AIDS no envelhecimento, bem como os diversos fatores que promovem a exposição desta população ao vírus, devido à falta de investigação sobre o assunto (Santos \& Assis, 2011).

Torna-se, então, importante, subsidiar o conhecimento dos profissionais de saúde acerca do HIV/AIDS na população que está envelhecendo de modo a promover a otimização da assistência a essa população. Espera-se que este estudo possa contribuir para a abordagem multiprofissional ao indivíduo, fornecendo subsídios para o desenvolvimento de ações de cunho preventivo a fim de qualificar a atenção à saúde.

Diante do que foi exposto, esta pesquisa possui como questão norteadora: como se apresenta a evolução dos casos de HIV/AIDS em pessoas com 50 anos ou mais? Para responder a tal questionamento, a pesquisa possui como objetivo analisar a evolução dos indivíduos acima de 50 anos ou mais com diagnóstico de HIV/AIDS atendidos em um Hospital universitário do Nordeste do Brasil. 


\section{Metodologia}

Trata-se de um estudo epidemiológico descritivo, documental, com abordagem quantitativa, de uma série histórica no período de 2006 a 2016, que examina a incidência (casos novos) e/ou a prevalência (casos existentes) de uma doença ou condição relacionada à saúde variando de acordo com determinadas características, como sexo, idade, escolaridade, renda, a ocorrência da doença/condição relacionada à saúde segundo o tempo, lugar ou pessoa.

Quanto à sua natureza, o estudo é caracterizado como quantitativo, pois lida com fatos e análises de dados estatísticos, e por descrever características de uma população (pessoas com 50 anos ou mais) ou fenômeno (infecção pelo HIV/Aids), a partir da relação entre variáveis selecionadas, é considerado como descritivo e ecológico (Menezes, Duarte, Carvalho, \& Souza, 2019; Pereira, Shitsuka, Parreira, \& Shitsuka, 2018).

Após autorização da instituição para a realização da pesquisa, iniciou-se a coleta de dados nos meses de janeiro a fevereiro/2017 através do banco de dados do Sistema Nacional de Agravos de Notificação (SINAN), disponível no Núcleo Hospitalar de Epidemiologia do Hospital Universitário Professor Alberto Antunes (HUPAA). Os pesquisadores selecionaram os dados necessários para atingir o objetivo dessa pesquisa, sendo utilizados os registros inerentes, referentes a evolução do HIV/AIDS entre as pessoas acima de 50 anos, levando em consideração as seguintes variáveis: idade; sexo; raça; escolaridade; procedência; ocupação; provável via de infecção e manifestações clínicas mais frequentes da doença, segundo os dois critérios de definição de casos de AIDS, denominados de "Rio de Janeiro/Caracas" que estabelece sendo todo caso de AIDS, para fins de vigilância epidemiológica, de acordo com a escala de sinais, sintoma ou doenças (Ministério da Saúde, 1998).

Após essa análise, os dados foram descritos através da estatística descritiva em forma de gráficos e tabelas, utilizando números absolutos e percentual.

\section{Resultados}

No período investigado, foram notificados 65 casos de infecção por HIV/AIDS entre pessoas com 50 anos ou mais atendidos em um hospital universitário do Nordeste. Entre os investigados, 41 (63,07\%) eram do sexo masculino e 24 (36,92\%) do sexo feminino. A maioria dos participantes se identificou como pertencente a raça parda 34 (52,3\%), seguida pelas raças com menor proporção, branco 14 (21,5\%) e preto $12(18,4 \%)$. A idade dos indivíduos de o maior número de notificações foi no grupo etário entre 50 e 59 anos com 54 (83,07\%). No tocante à escolaridade, 32,3\% possuíam ensino fundamental e em 27,7\% das fichas a escolaridade dos indivíduos foi ignorada, como pode ser observado na Tabela 1. 
Tabela 1. Casos de HIV/Aids em indivíduos com 50 anos de idade e mais (n=65), segundo variáveis faixa etária e escolaridade em Hospital Universitário do Nordeste, Maceió/AL, 2017.

\begin{tabular}{|c|c|c|c|c|c|c|}
\hline \multirow{2}{*}{ Variáveis } & \multicolumn{2}{|c|}{ Masculino } & \multicolumn{2}{|c|}{ Feminino } & \multicolumn{2}{|c|}{ Total } \\
\hline & $\mathrm{n}$ & $\%$ & $\mathrm{n}$ & $\%$ & $\mathrm{n}$ & $\%$ \\
\hline \multicolumn{7}{|l|}{ Faixa Etária (anos) } \\
\hline $50-59$ anos & 32 & 78,04 & 22 & 91,6 & 54 & 83,07 \\
\hline $60-69$ anos & 7 & 17,07 & 2 & 8,3 & 9 & 13,84 \\
\hline$\geq 70$ anos & 2 & 4,87 & - & - & 2 & 3,07 \\
\hline \multicolumn{7}{|l|}{ Escolaridade } \\
\hline Analfabeto & 6 & 14,63 & 6 & 25 & 12 & 18,46 \\
\hline Fundamental & 13 & 31,7 & 8 & 33,3 & 21 & 32,3 \\
\hline Médio & 6 & 14,63 & 3 & 12,5 & 9 & 13,84 \\
\hline Superior & 3 & 7,31 & 2 & 8,3 & 5 & 7,7 \\
\hline Ignorado & 13 & 31,7 & 5 & 20,8 & 18 & 27,7 \\
\hline Total & 41 & 100 & 24 & 100 & 65 & 100 \\
\hline
\end{tabular}

Fonte: Banco de dados do Núcleo Hospitalar de Epidemiologia - HUPAA.

Em relação à procedência, mais que a metade era residente da capital, com $42(64,6 \%)$. Os demais eram provenientes de outros municípios. Com relação a categoria orientação sexual foi identificada sete casos (10,7\%) bissexuais, presentes apenas no sexo masculino. Vale ressaltar dentro desta perspectiva 11 notificações estavam com os dados ignorados, isso nos remete a falha no preenchimento das fichas de notificações, desta forma tornando os dados limitados, conforme demonstra a Tabela 2.

Tabela 2. Casos de HIV/Aids em indivíduos com 50 anos de idade e mais, segundo variáveis de procedência e provável via de exposição em Hospital Universitário do Nordeste, Maceió/AL, 2017.

\begin{tabular}{|c|c|c|c|c|c|c|}
\hline \multirow{2}{*}{ Variáveis } & \multicolumn{2}{|c|}{ Masculino } & \multicolumn{2}{|c|}{ Feminino } & \multicolumn{2}{|c|}{ Total } \\
\hline & $\mathbf{n}$ & $\%$ & $\mathbf{n}$ & $\%$ & $\mathbf{n}$ & $\%$ \\
\hline \multicolumn{7}{|c|}{ Procedência (Nº de habitantes) } \\
\hline Capital & 24 & 58,53 & 18 & 75 & 42 & 64,6 \\
\hline Interior & 17 & 41,42 & 6 & 24,96 & 18 & 32,29 \\
\hline \multicolumn{7}{|l|}{ Provável via de exposição } \\
\hline Heterossexual & 20 & 48,78 & 23 & 95,8 & 43 & 66,15 \\
\hline Homossexual & 4 & 9,75 & - & - & 4 & 6,15 \\
\hline Bissexual & 7 & 17,07 & - & - & 7 & 10,76 \\
\hline Ignorado & 10 & 24,39 & 1 & 4,16 & 11 & 16,92 \\
\hline Total & 41 & 100 & 24 & 100 & 65 & 100 \\
\hline
\end{tabular}

Fonte: Banco de dados do Núcleo Hospitalar de Epidemiologia - HUPAA.

No que se refere à ocupação por gênero, dentre o sexo feminino a ocupação mais frequente foi "do lar” com $12,3 \%$ dos registros, já entre os indivíduos do sexo masculino houve o predomínio de aposentados (9,2\%). Vale ressaltar que em 46,1\% das fichas de notificações, esta variável foi ignorada, onde novamente nos remeta o descompromisso no preenchimento das fichas de notificações.

Ao analisarmos a série histórica dos números de casos de HIV/AIDS de acordo com o ano de notificação de cada um dos indivíduos com 50 anos ou mais nos anos investigados, observou-se que a curva de incidência se revelou estável entre os 
anos de 2007 e 2010, observando um aumento gradativo no número de notificações a partir do ano de 2012 . Contudo, é importante ressaltar que não foram notificados casos de HIV/AIDS em indivíduos com 50 anos ou mais nos anos de 2006 e 2012 , justifica-se pelo motivo que nos anos de 2006 a 2012 estava sendo implantado no hospital o processo de notificação epidemiológica, desta forma este fato explica os baixos números de notificações neste período, sendo demonstrado na Figura 1.

Figura 1. Casos de HIV/AIDS em indivíduos com 50 anos ou mais (n=65), segundo ano de notificação em hospital universitário de nordeste, Maceió/AL, 2017.

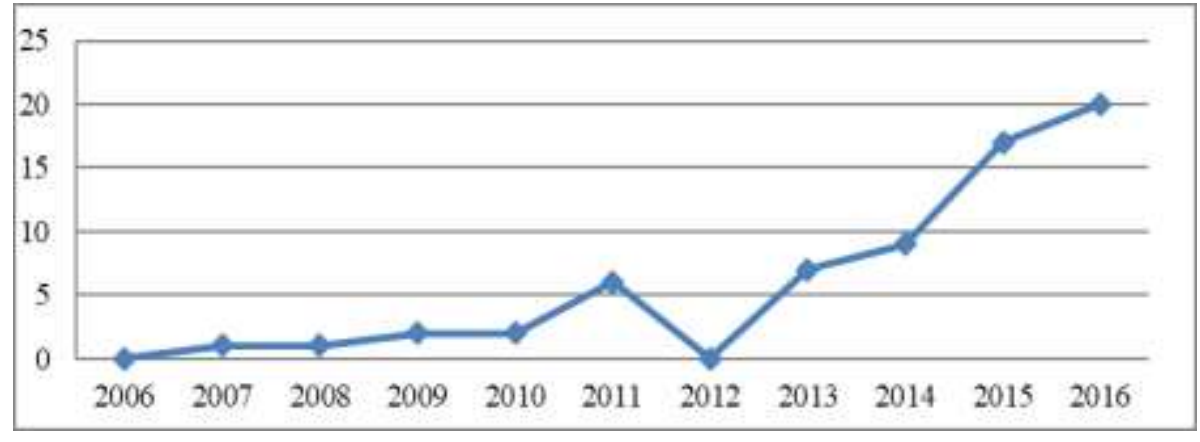

Fonte: Banco de dados do Núcleo Hospitalar de Epidemiologia - HUPAA.

Com relação às manifestações clínicas, de acordo com critério de definição de casos de AIDS 'Rio de Janeiro-Caracas’, as mais frequentes foram: caquexia $(36,9 \%)$, astenia $(27,6 \%)$, diarreia $(24,6 \%)$ e febre $(21,5 \%)$. Outras manifestações foram observadas em menores proporções, dentre elas: tosse persistente $(16,9 \%)$, anemia/linfopenia $(15,3 \%)$ e candidíase oral (n=9; 13,8\%). Dos 65 casos, 7 idosos evoluíram para óbito, em sua maioria em detrimento da AIDS (n=6), e em 58 casos os pacientes deram seguimento ao tratamento, após o diagnóstico.

\section{Discussão}

A propagação do HIV/AIDS no Brasil vem evidenciando mudanças no seu perfil, descaracterizando os grupos de riscos para este agravo e atingindo qualquer indivíduo da sociedade, em especial os mais vulneráveis (Souza et al., 2021).

O resultado desse estudo demonstrou que o perfil da população mais afetado pelo HIV/AIDS são os de homens de idade entre 50 a 59 anos, o que difere da literatura, pois a faixa mais acometida encontra-se na idade de 60 anos ou mais. Esse dado nos leva a inferir que pelo menos uma parte da população estudada foi infectado de 5 a10 anos atrás, quando se encontravam na fase adulta, uma vez que o processo de adoecimento leva um tempo entre a suspeita e a confirmação da situação de soropositivo.

Ao analisar as informações referentes aos dados sociodemográficos dos indivíduos, verificou-se que a maioria possui até 8 anos de estudo, destacando que 18,46\% são analfabetos, caracterizando um fenômeno presente nesse agravo, a pauperização. Demonstrando que as populações já tradicionalmente marginalizadas estão cada vez mais se infectando pelo HIV/AIDS. Como dificuldade, acrescenta-se o difícil acesso aos serviços de saúde e à informação (Andrade et al., 2017; Silva et al., 2018; Braga, Franzmann \& Fontenele, 2020; Souza et al., 2021).

Outro dado sociodemográfico é a procedência, onde se observa que o maior número de notificações da doença está presente na capital, esta informação vem a confirmar que o HIV/AIDS é um agravo típico dos grandes centros urbanos. Porém, também, observa-se um número elevado de notificações nas cidades do interior, apresentando um novo processo o de interiorização. Este fato só vem reafirmar uma nova tendência que se mostra nos últimos anos do HIV/AIDS, demonstrado em outras pesquisas (Braga et al., 2020; Amorim et al., 2019) 
Outra variável importante a ser considerada é a orientação sexual, por ser relevante ao determinar a provável via de exposição à doença, no qual pode-se verificar o processo de heterossexualização, característica que não diferente de outras faixas etárias. Autores relatam, o que vem contribuindo para esse novo perfil do HIV/AIDS é o aumento da atividade sexual entre indivíduos com 50 anos ou mais, a disposição de tecnologia que melhora e prolonga a desempenho sexual, e a resistência em usar meios de prevenção. Além disso, a falsa crença da assexualidade nessa faixa etária desencadeia graves consequências, e os profissionais de saúde continuam não abordando a prática sexual entre eles (Autenrieth et al., 2018; Barros, Miranda \& Coelho, 2018; Silva et al., 2018; Capellari et al., 2019; Braga et al., 2020; Brandão, Angelim, Marques, Oliveira \& Abrão, 2020).

Entre as principais formas de exposição do HIV/AIDS no sexo masculino são observadas nas opções homo-bissexuais, demonstrando que o HIV/AIDS traz à tona a discussão de preconceitos enraizados na sociedade, onde as escolhas que as pessoas fazem em relação à forma de manifestar-se sexualmente são fortemente influenciadas pelas questões de gênero, uma construção sociocultural com papeis sociais pré-estabelecidos. Por outro lado, notamos a feminização da epidemia, encontramos mulheres que se sentem constrangidas em ter de pedir ao companheiro que use preservativo e homens com receio de perder a ereção, pondo, assim, em risco a relação sexual (Lima \& Souza, 2018; Suto et al., 2020).

Esses fatores vêm a contribuir para o aumento da incidência do HIV/AIDS nos indivíduos com 50 anos ou mais. Que pode ser observado na análise da evolução temporal do número de casos de HIV/AIDS, verificando-se um aumento gradativo a partir do ano 2012. Nos anos anteriores apresentaram uma baixa incidência, isto ocorreu pelo motivo do serviço de notificação de casos estava em processo de implantação no hospital no período de 2006 a 2011.

Diante disso, percebe-se, epidemiologicamente, a relevância do HIV/AIDS na população com 50 anos ou mais, não pelos números absolutos, mas pelo aumento expressivo da doença nesta população a cada ano. O que diverge em relação à estabilização e/ou redução da incidência nos últimos anos evidenciado nos outros grupos de faixa etária. Este fato vem corroborar com os números já identificados em outros estudos estatísticos realizados no Brasil (Oliveira, Paz \& Melo, 2013; Jesus et al., 2014; Silva et al., 2018; Souza et al., 2021)

Entre as variáveis analisadas, observa-se que a maioria dos indivíduos apresentou mais de um tipo de manifestação clínica. E um dado relevante que a pesquisa demonstra é que, entre os casos, apresentaram óbitos principalmente por AIDS. Desta forma, o HIV/AIDS por ser considerada uma condição crônica e apresenta manifestações clínicas sucessivas e/ou simultâneas, e incluindo os efeitos colaterais da terapia antirretroviral que o seguirá pelo resto da vida. Faz necessário que os indivíduos sejam avaliados de forma sistematizada e contínua, com enfoque na capacidade funcional, por ser realizada com portadores de HIV/AIDS. Vale ressaltar que o desfecho epidemiológico dos portadores de HIV é diferente dos pacientes com Aids, e que a idade é um fator importante a considerar diante desse perfil (Silva et al., 2018; Souza et al., 2021; Vieira, Costa, Dias, Araújo, \& Gazila, 2021).

Cuidar de uma pessoa que está no processo de envelhecimento exige preparo e competência da equipe de saúde. Se o indivíduo é portador de HIV/AIDS, a situação se agrava, na medida em que traz consigo fatores que acentua a gravidade de sua saúde, necessitando de um atendimento especializado, não somente pela sua condição sorológica, mas também pelos fatores sociais, econômicos e ambientes que por conta do envelhecimento está agravada. Sendo assim, tem-se a necessidade de políticas públicas que atendam a população envelhecida, justificando-se por ser um grupo que enfrenta situações de conflitos, falta de perspectivas concretas no cotidiano, além das dificuldades para manter o tratamento, principalmente quanto aspectos referentes ao gênero, onde a mulher surge como a parte submissa no relacionamento, favorecendo a vulnerabilidade à infecção pelo HIV (Santos \& Assis, 2011; Braga et al., 2020; Suto et al., 2020).

O estudo, porém, apresentou uma limitação referente aos dados ilegíveis ou ao não preenchimento por parte dos profissionais. Essas limitações apontam que o uso de dados secundários não permite ao pesquisador controlar possíveis erros decorrentes do registro ou a falta do preenchimento de variáveis nas fichas, além de prováveis subnotificações. Como é o caso 
da variável ocupação que em $46,1 \%$ das fichas este dado foi negligenciado. Todavia, acredita-se que por ser tratar de dados locais oficiais e de preenchimento obrigatório, seus resultados permitiram alcançar o objetivo proposto.

\section{Considerações Finais}

O estudo demonstrou um aumento progressivo para evolução dos indivíduos com 50 anos ou mais que apresentam o diagnóstico de HIV/AIDS atendidos em um Hospital universitário. E foi possível observar, ainda, os processos de heterossexualização, pauperização e uma crescente interiorização dos casos de HIV/AIDS entre os indivíduos com 50 anos ou mais.

Desta forma, assistir aos indivíduos que estão em processo de envelhecimento, que se apresentam sexualmente ativos e cercados por preconceitos, exige a atitude de profissionais cada vez mais preparados, abertos a novos paradigmas e com conhecimento das particularidades apresentadas por eles. Assim, deve-se estar preparado para oferecer uma abordagem segura e responsável aos clientes, principalmente no que tange à sexualidade e, consequentemente, agir prevenindo doenças evitáveis, o que parece ser um grande desafio para os profissionais de saúde.

Sugere-se à comunidade científica que continue a produzir análises estatísticas com dados atualizados disponíveis, realizando comparativo entre as publicações mais recentes, estudos previamente elaborados e que trace o perfil das pessoas que vivem com o HIV, em especial àquelas com idade igual ou superior a 50 anos.

\section{Referências}

Amorim, P. J. F., Abreu, I. M. de, Mendes, P. M., Moura, M. Á. P., Araújo, T. M. E. de, \& Falcão, L. M. (2019). Perfil sociodemográfico e a evolução clínica dos pacientes com síndrome da imunodeficiência humana. Rev enferm UFPE on line, 13, e241310. https://doi.org/10.5205/1981-8963.2019.241310.

Andrade, J., Ayres, J. A., Alencar, R. A., Duarte, M. T., \& Parada, C. M. (2017). Vulnerabilidade de idosos a infecções sexualmente transmissíveis. Acta Paul Enferm, 30(1), 8-15.

Autenrieth, C. S., Beck, E. J., Stelzle, D., Mallouris, C., Mahy, M., \& Ghys, P. (2018). Global and regional trends of people living with HIV aged 50 and over: Estimates and projections for 2000-2020. PLOS ONE, 13(11), e0207005. https://doi.org/10.1371/journal.pone.0207005.

Barros, T. S., Miranda, K. C. L, \& Coelho, M. M. F. (2018). Idosos com HIV/AIDS: compreendendo a base ideológica de suas vivências. Rev enferm UERJ, 1:e12978. http://dx.doi.org/10.12957/reuerj.2018.12978.

Braga, M. I. A., Franzmann, U. T., \& Fontenele, R. M. (2020). Produção do conhecimento sobre a vulnerabilidade dos idosos quanto à exposição ao HIV/AIDS. Research, Society and Development, 9(11), e3789119989. http://dx.doi.org/10.33448/rsd-v9i11.9989.

Brandão, B. M. G. M., Angelim, R. C. M., Marques, S. C., Oliveira, R. C., \& Abrão, F. M. S. (2020). Convivendo com o HIV: estratégias de enfrentamento de idosos soropositivos. Rev Esc Enferm USP, 54:e03576. https://doi.org/10.1590/S1980-220X2018027603576.

Capellari, B., Vidor, A. O., Pezzini, M. F., Godoy, S., Maffei, V., \& Poeta, J. Conhecimento sobre HIV/aids segundo idosos de cidades da Serra Gaúcha/RS. (2019). Revistas da USP Medicina (Ribeirão Preto) [Online] 52(4), 305-312. Retrieved from https://www.revistas.usp.br/rmrp.

Instituto Brasileiro de Geografia e Estatística [IBGE]. (2019). Longevidade: viver bem e cada vez mais. Retratos a revista do IBGE, (16), 1-28. https://agenciadenoticias.ibge.gov.br/media/com_mediaibge/arquivos/d4581e6bc87ad8768073f974c0a1102b.pdf.

Jesus, S. M. C. de, Caldas, A. J. M., Côrrea, R. G. C. F., Soares, D. L., Pereira, L. F. B., \& Aquino, D. M. C. de. (2014). Rev Pesq Saúde, 15(2), 276-9. http://www.periodicoseletronicos.ufma.br/index.php/revistahuufma/article/view/3264.

Lima, I. S., \& Souza, F. M. A. de (2018). AIDS e envelhecimento. Revista Eletrônica Cosmopolita em Ação, 5(2), 01-32.

Menezes, A. H. N., Duarte, F. R., Carvalho, L. O. R., \& Souza, T. E. S. (2019). Metodologia científica: teoria e aplicação na educação a distância. PetrolinaPE. https://portais.univasf.edu.br/dacc/noticias/livro-univasf/

Ministério da Saúde. Secretaria de Políticas de Saúde. Coordenação Nacional de DST e Aids. (1998). Revisão da definição nacional de casos de aids em indivíduos com 13 anos ou mais, para fins de vigilância epidemiológica. Brasília(Brasil): Ministério da Saúde. 36p. http://bvsms.saude.gov.br/bvs/publicacoes/074_02revisao.pdf

Oliveira, M. L. C. de, Paz, L. C., \& Melo, G. F. de. (2013). Dez anos de epidemia do HIV-AIDS em maiores de 60 anos no Distrito Federal - Brasil. Rev Bras Epidemiol [online] 16(1), 30-9. http://dx.doi.org/10.1590/S1415-790X2013000100003.

Pereira, A. S., Shitsuka, D. M., Parreira, F. J., \& Shitsuka, R. (2018). Metodologia da pesquisa científica. UFSM. https://repositorio.ufsm.br/. 
Research, Society and Development, v. 10, n. 4, e6110413852, 2021

(CC BY 4.0) | ISSN 2525-3409 | DOI: http://dx.doi.org/10.33448/rsd-v10i4.13852

Pourcher, V., Goumelen, J., Bureau, I., \& Bouee, S. (2020). Comorbidities in people living with HIV: An epidemiologic and economic analysis using a claims database in France. PLoS ONE, 15(12): e243529. https://doi.org/10.1371/journal.pone.0243529.

Santos, A. F. M., Assis, M. de. (2011). Vulnerabilidade das idosas ao HIV/AIDS. Rev Bras Geriatr Gerontol, 14(1), 147-57. http://www.scielo.br/pdf/rbgg/v14n1/a15v14n1.pdf.

Silva, B. N. da, Sarmento, W. M., Silva, F. C., Pereira, M. G., Silva, C. R. D. V., \& Véras, G. C. B. (2018). Panorama epidemiológico da AIDS em idosos. Hygeia Revista Brasileira de Geografia Médica e da Saúde, 14(29), 80-88. http://www.seer.ufu.br/index.php/hygeia.

Souza, E. V., Jr., Cruz, D. P., Caricchio, G. M. N., Jesus, M. A. S. de, Boery, R. N. S. O., \& Boery, E. N. (2021). Aspectos epidemiológicos da morbimortalidade pelo vírus da imunodeficiência humana no nordeste brasileiro. Rev Fund Care Online. 13, 144-149. https://dx.doi.org/10.9789/2175-5361.rpcfo.v13.8025.

Suto, C. S. S., Coelho, E. A. C., Paiva, M. S., Porcino, C., Cabral, L. S., \& Marques, S. C. (2020). Women of different generations living with HIV: social representations about sexuality. Rev Esc Enferm USP, 54: e03658. https://doi.org/10.1590/S1980-220X2019018303658.

Vieira, C. P. B., Costa, A. C. S. S., Dias, M. C. L., Araújo, T. M. E. de, \& Gazila, F. T. (2021). Tendência de infecções por HIV/Aids: aspectos da ocorrência em idosos entre 2008 e 2018. Esc Anna Nery, 25(2), e20200051. https://doi.org/10.1590/2177-9465-EAN-2020-0051. 